\title{
Similaridade morfológica entre turdídeos sintópicos em um parque urbano na Região Sul do Brasil
}

Huilquer Francisco Vogel ${ }^{1 *}$, João Marcelo Deliberador Miranda ${ }^{2}$, Cláudio Henrique Zawadzki ${ }^{3} \&$ Rafael Metri $^{4}$

${ }^{1}$ Programa de Pós-graduação em Ciências Ambientais, Universidade Estadual de Maringá, Bloco G-90, Sala 16, Avenida Colombo, 5790, Centro, 87020-900, Maringá, Paraná, Brasil.

${ }^{2}$ Departamento de Ciências Biológicas, Universidade Estadual do Centro-Oeste, campus Cedeteg, Rua Simeão Camargo Varela de Sá, 3, Caixa Postal 3010, 85040-080, Guarapuava, Paraná, Brasil.

${ }^{3}$ Núcleo de Pesquisas em Limnologia, Ictiologia e Aquicultura, Departamento de Biologia, Universidade Estadual de Maringá, Bloco G-90, Sala 18B, Avenida Colombo, 5790, Centro, 87020-900, Maringá, Paraná, Brasil.

${ }^{4}$ Departamento de Ciências Biológicas, Universidade Estadual do Paraná, Campus FAFIPAR, Rua Comendador Correia Junior, 117, 83203-280, Paranaguá, Paraná, Brasil.

\begin{abstract}
Resumo - A sobreposição morfológica é um importante atributo para a interpretação de processos de competição interespecífica. Neste sentido, o objetivo deste trabalho foi avaliar o grau de sobreposição morfológica entre cinco espécies de turdídeos (Turdus rufiventris, T. albicollis, T. leucomelas, T. amaurochalinus e T. subalaris) sintópicos em um fragmento urbano de Floresta Ombrófila Mista Montana no município de Guarapuava, Centro-sul do Paraná. As aves foram capturadas em redes de neblina. Aferições biométricas foram realizadas nos exemplares. Posteriormente, foram efetuadas análises de similaridade e ordenações multivariadas. A análise MANOVA indicou uma separação morfológica entre as espécies $F_{(24,482)}=17,8 ; P<0,0001$. Entretanto, o teste post hoc indicou que não houve separação estatística entre T. albicollis e T. subalaris. Os resultados do índice de similaridade indicaram que as espécies mais semelhantes desta taxocenose são T. leucomelas e T. rufiventris $(0,987)$. Turdus albicollis e $T$. subalaris também apresentaram alta similaridade $(0,983)$. Apesar das espécies serem muito semelhantes morfologicamente, existem indicativos de mecanismos ecológicos que permitem a coexistência delas, como padrões distintos de ocorrência e utilização diferenciada do hábitat.
\end{abstract}

Palavras-chave adicionais: biometria, coexistência, Turdus.

\begin{abstract}
Morphological similarity between syntopic thrushes in an urban park in southern Brazil) - Morphological overlap is an important attribute to understand processes of interspecific competition. In this sense, the objective of this study was to evaluate the degree of morphological overlap between five syntopic thrushes species (Turdus rufiventris, $T$. albicollis, T. leucomelas, T. amaurochalinus and T. subalaris) in an urban fragment of Araucaria Forest in Guarapuava, central-southern region of Paraná State. Birds were captured through mist-nets. Biometric measurements were performed in the birds and subsequently analyses of similarity and multivariate ordinations were also performed. The MANOVA analysis indicated separation among species $F_{(24,482)}=17.8 ; P<0.0001$. However, post hoc test showed no statistical separation between $T$. albicollis and T. subalaris. The similarity index results indicated that the most similar species that integrate the taxocenose are T. leucomelas and T. rufiventris (0.987). Turdus subalaris and T. albicollis also showed high similarity values $(0.983)$. Although the species are morphologically very similar, there are indications of ecological mechanisms that allow their coexistence, such as distinct patterns of occurrence and differential use of habitat.
\end{abstract}

Additional key words: biometry, coexistence, Turdus.

A família Turdidae (Aves: Passeriformes) possui aproximadamente 300 espécies e destaca-se por sua ampla distribuição, ocorrendo nas Américas, África e Eurásia (Clement 2000; Collar 2005). No Brasil, são registradas 18 espécies de turdídeos (CBRO 2011; O’Neill et al. 2011). De modo geral, as espécies deste grupo são excelentes modelos para estudos ecológicos, principalmente devido à ocorrência e abundância, tanto em ambientes urbanos quanto naturais. A família apresenta tanto espécies susceptíveis quanto resistentes às modificações ambientais (Vogel 2012). Contudo, poucos são os estudos que buscam esclarecer como são estruturadas as taxocenoses de turdídeos no Brasil (Sick

*Autor para correspondência: huilquer@hotmail.com

Editor responsável: Caio Graco Machado

Submetido em: 15 jun. 2012; publicação eletrônica: 19 dez. 2012
1997).

Apesar de existirem dados biométricos destas espécies na literatura (e.g., Sick 1997; Clement 2000; Ridgely \& Tudor 2001; Collar 2005; Sigrist 2006), seus valores geralmente são de baixa precisão, sendo expressos em números inteiros, sobre os quais não é possível detalhar a variação do tamanho dos espécimes. Esses valores são muito divergentes e, algumas vezes, aproximam morfologicamente espécies que, conhecidamente, apresentam divergências morfológicas evidentes.

Tratando-se de estudos bioecológicos de turdídeos no Brasil, alguns autores deram importantes contribuições, principalmente no campo da biologia da alimentação (e.g., Leite et al. 2010; Sazima \& D'Angelo 2011) e da parasitologia 
(e.g., Storni et al. 2005; Enout et al. 2009). Segundo Sick (1997), processos que permitem a coexistência entre turdídeos não são amplamente estudados, evidentes ou facilmente compreensíveis. Evidências indicam que tal coexistência envolve a partilha dos mesmos recursos em proporções diferenciadas e ocupação diferencial do hábitat (Gasperin \& Pizo 2009; Vogel et al. 2011).

A detecção de diferenças sutis na morfologia de espécies consideradas morfologicamente semelhantes pode ser útil para a compreensão dos fatores que permitem a coexistência delas em um dado ambiente (MacArthur \& Levins 1967). Evidências indicam que modificações morfológicas podem garantir leve distinção na ocupação de recursos. Por sua vez, esta distinção permite que, na relação de espécies coocorrentes, haja uma sobreposição de nichos, porém não total, principalmente se os recursos forem limitantes (Wiens 1982; Putmam 1996). Assim, trabalhos que levem em consideração a influência da forma em processos de segregação ecológica podem ser úteis na interpretação de processos de exclusão competitiva entre espécies aparentadas e coocorrentes, sobretudo em espécies que partilham nicho trófico semelhante (MacArthur \& Levins 1967). Neste sentido, o objetivo deste trabalho foi analisar a sobreposição morfológica entre cinco espécies de turdídeos ocorrentes em um parque urbano em Guarapuava, Centro-Sul do estado do Paraná.

\section{Materiais e Métodos}

Área de estudo. $\mathrm{O}$ estudo foi realizado no Parque $\mathrm{Mu}$ nicipal das Araucárias (PMA; 2521' 03”'S, 51²8'01,9”W), município de Guarapuava, mesorregião Centro-Sul do estado do Paraná (Figura 1). A área de estudo possui aproximadamente 20 ha cobertos por Floresta Ombrófila Mista Montana (Floresta com Araucárias), com altitude em torno de $1070 \mathrm{~m}$. O PMA encontra-se delimitado na parte norte pelo rio Xarquinho, onde uma zona de formação florestal em estádio inicial de sucessão secundária faz transição com uma área de matriz agrícola (Cordeiro \& Rodrigues 2007). O clima possui temperatura média anual de $16,8^{\circ} \mathrm{C}$ e um regime pluviométrico próximo de $1.800 \mathrm{~mm}$ anuais, caracterizando-se como tipo $\mathrm{Cfb}$ (temperado úmido com verão quente), segundo a classificação de Köppen-Geiger (Thomaz $\&$ Vestena 2003).

Coleta de dados. Os dados de campo foram obtidos ao longo de 12 meses (de dezembro de 2008 a novembro de 2009). A captura das aves foi realizada utilizando seis redes de neblina $6 \times 3 \mathrm{~m}$ ( 3 redes com malha $20 \mathrm{~mm}$ e 3 de 35 $\mathrm{mm}$ ), dispostas na área de estudo a uma altura de 50-150 $\mathrm{cm}$ do solo. As redes foram dispostas em locais previamente selecionados, de acordo com a observação da atividade das aves, constatada em um dia anterior, com cuidado para contemplar igualmente ambientes de borda e o interior do fragmento amostrado, maximizando desta forma o sucesso de captura. As redes permaneceram abertas das $7 \mathrm{~h} 00$ às $19 \mathrm{~h} 00$, quatro dias por mês, totalizando $3.456 \mathrm{~h} /$ rede, um esforço amostral de $7488 \mathrm{~h} \mathrm{~m}^{2}$ (Straube \& Bianconi 2002).

Os indivíduos capturados foram anilhados e classificados como adultos, ninhegos ou juvenis, de acordo com a textura do tarso, dureza das unhas, plumagem e ossificação craniana (CEMAVE 2008). Aves em processo de muda de penas foram desconsideradas para as análises durante aquela amostragem. Foram aferidas as seguintes variáveis biométricas: 1- massa corpórea (g); 2- comprimento total ( $\mathrm{cm}$ ou $\mathrm{mm}$ ); 3- comprimento do bico (mm); 4- tarso (mm); 5- asa (mm); e 6- cauda ( $\mathrm{mm})$. Essas medidas foram obtidas com paquímetro digital (precisão de $0,01 \mathrm{~mm}$ ) e balança analógica (precisão de $0,1 \mathrm{~g}$ ). Os parâmetros unilaterais e bilaterais mensurados foram padronizados do lado direito das aves, evitando efeitos de assimetria, de modo que as medidas foram realizadas com base em Sick (1997) e Auricchio (2001).

Análise de dados. Os caracteres morfológicos foram submetidos a uma análise de componentes principais, utilizando o critério scree plot para retenção dos eixos, demonstrando a participação das variáveis para a segregação morfológica entre as espécies. Uma análise de similaridade (Cluster) de Bray-Curtis foi efetuada com base no conjunto de médias dos caracteres biométricos avaliados, mensurando o nível de sobreposição morfológica entre diferentes espécies. Posteriormente, os dados biométricos foram comparados entre as espécies através de uma análise multivariada de variâncias (MANOVA) com teste post hoc (Hotelling's Tsquared distribution). Este teste teve por objetivo checar a possibilidade de variação no conjunto total de variáveis das espécies. Os dados também foram testados por meio de análise de variância (ANOVA one way), com nível de significância $(a=0,05$; teste post hoc LSD). As análises foram realizadas sem transformações dos dados, uma vez que as variáveis atenderam aos pressupostos de normalidade $\mathrm{e}$ homocedasticidade. Procedimentos analíticos foram efetuados no programa PAST $^{\odot}$, versão 2.16 (Hammer et al. 2001).

\section{Resultados}

Foram capturadas cinco espécies do gênero Turdus Linnaeus, 1758; 34\% dos exemplares de Turdus rufiventris Vieillot, $1818(\mathrm{n}=57) ; 31 \%$ de T. leucomelas Vieillot, 1818 $(\mathrm{n}=51) ; 27,5 \%$ de T. amaurochalinus Cabanis, $1850(\mathrm{n}=$ 44); 5\% de T. albicollis Vieillot, $1818(\mathrm{n}=8)$; e finalmente, $2,5 \%$ de T. subalaris (Seebohm, 1887) $(\mathrm{n}=4)$.

Através da ordenação da análise de componentes principais utilizando o critério de scree plot, apenas o PC1 foi retido. Contudo, as variáveis demonstraram-se fracamente correlacionadas com os eixos. Desta forma, são fornecidos os valores de correlações entre variáveis e os três primeiros eixos (Tabela 1) como informações complementares. Os resultados da análise de componentes principais (Figura 2a) 


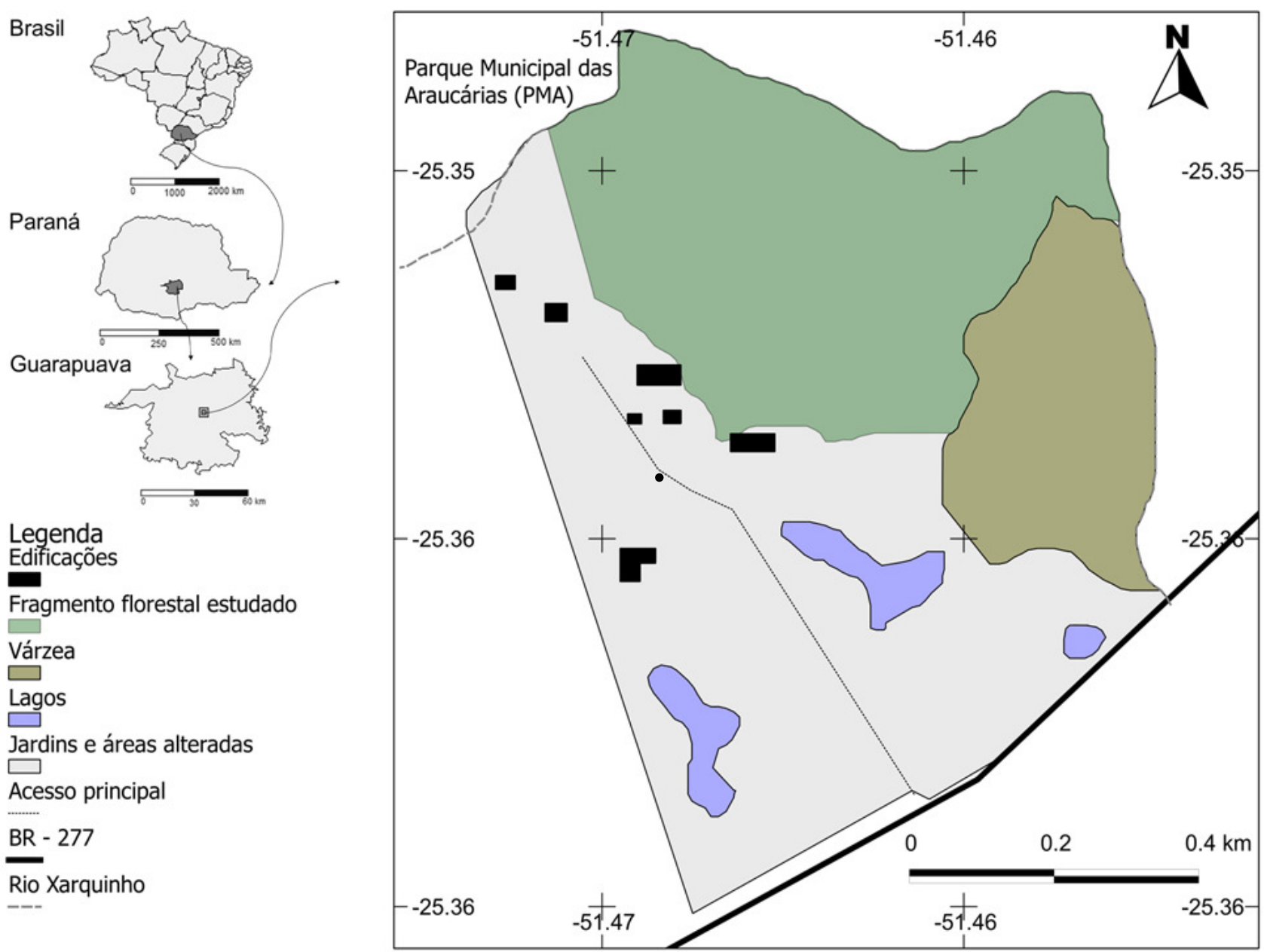

Figura 1. Mapa do Parque Municipal das Araucárias, município de Guarapuava, estado do Paraná, Brasil, demonstrando o fragmento florestal estudado e hábitats adjacentes.

permitiram constatar que a massa $(\mathrm{PC} 1, \mathrm{r}=0,43 ; \mathrm{PC} 2, \mathrm{r}=-$ $0,49)$, comprimento do bico (PC2, $r=0,66)$, tamanho da asa $(\mathrm{PC} 2, \mathrm{r}=0,56)$ e tarso $(\mathrm{PC} 3, \mathrm{r}=-0,48)$ são variáveis importantes para diferenciar as espécies.

Tabela 1. Correlações entre variáveis e eixos obtidas através da Análise de Componentes Principais entre as variáveis biométricas obtidas de cinco espécies de turdídeos.

\begin{tabular}{lccc}
\hline Variáveis & \multicolumn{3}{c}{ Eixos e correlações } \\
\cline { 2 - 4 } & $P C$ l & $P C 2$ & $P C 3$ \\
\hline Massa (g) & 0,43 & 0,02 & $-0,49$ \\
Comprimento (mm) & 0,09 & 0,03 & $-0,02$ \\
Comprimento do bico (mm) & 0,35 & $\mathbf{0 , 6 6}$ & 0,26 \\
Tarso (mm) & 0,41 & $-0,27$ & $-0,48$ \\
Asa (mm) & 0,38 & $\mathbf{- 0 , 5 6}$ & $-0,18$ \\
Cauda (mm) & 0,41 & $-0,37$ & 0,41 \\
Autovalores & 3,17 & 0,94 & 0,64 \\
\hline $\mathbf{\%} \boldsymbol{\sigma}^{\mathbf{2}}$ & 52,87 & 15,74 & 10,79 \\
\hline$\sum \% \boldsymbol{\sigma}^{\mathbf{2}}$ & 52,87 & 68,61 & 78,40 \\
\hline
\end{tabular}

A análise de Cluster (Figura 2b) mostrou as maiores similaridades morfométricas entre T. leucomelas e T. rufiventris $(0,987)$ e $T$. subalaris e $T$. albicollis $(0,983)$. As espécies mais divergentes foram $T$. rufiventris e T. subalaris $(0,940)$.

O resultado da comparação morfométrica demonstrou diferenças significativas entre as espécies para a massa $\left(F_{(4,144)}\right.$ $=45,23 ; P<0,01)$; para o comprimento total, novamente houve diferença entre as espécies $\left(F_{(4,144)}=14,53 ; P<0,01\right)$. De modo semelhante, variáveis de comprimento do bico $\left(F_{(3,139)}=22,85 ; P<0,01\right)$, tarso $\left(F_{(4,144)}=45,23 ; P<0,01\right)$, asa $\left(F_{(4,144)}=10,23 ; P<0,01\right)$ e cauda $\left(F_{(4,144)}=14,53 ; P<\right.$ $0,01)$, novamente apresentaram divergência estatística. As médias dos dados morfológicos, assim como as divergências, mínimas e máximas de cada variável entre as espécies podem ser observadas na Tabela 2. A análise MANOVA indicou separação morfológica entre as espécies $\left(F_{(24,482)}=\right.$ $17,8 ; P<0,0001)$. Contudo, testes a posteriori indicaram que não houve separação estatística entre Turdus albicollis e Turdus subalaris. 
(a)

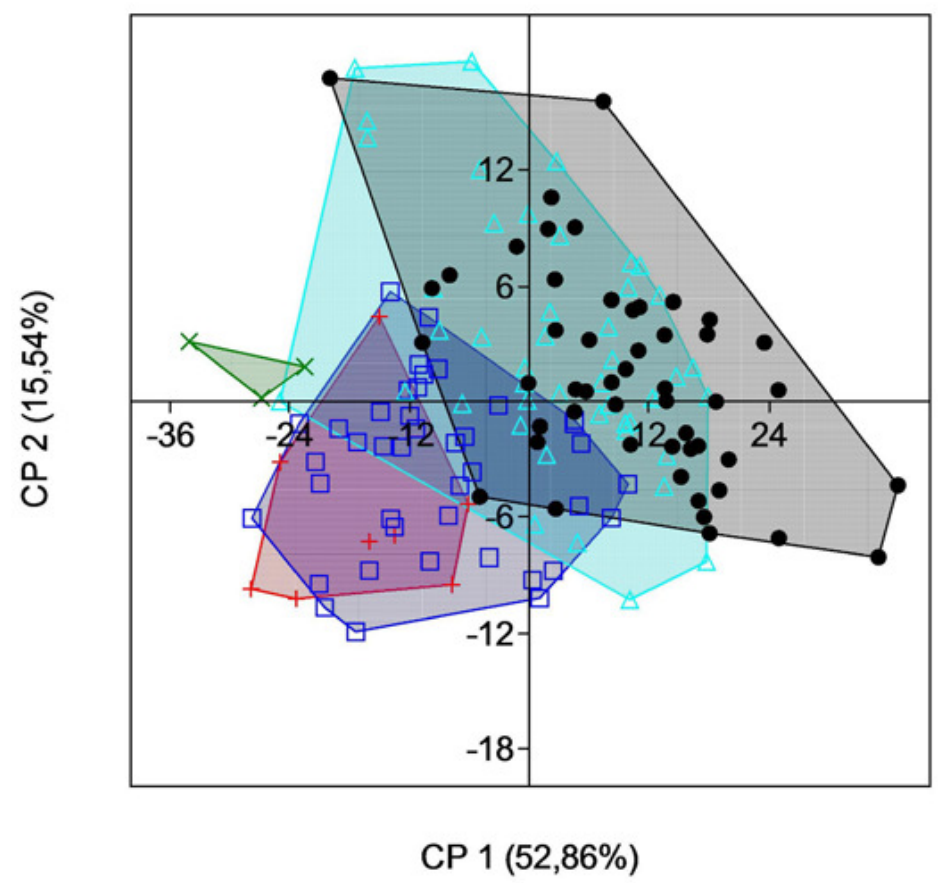

(b)

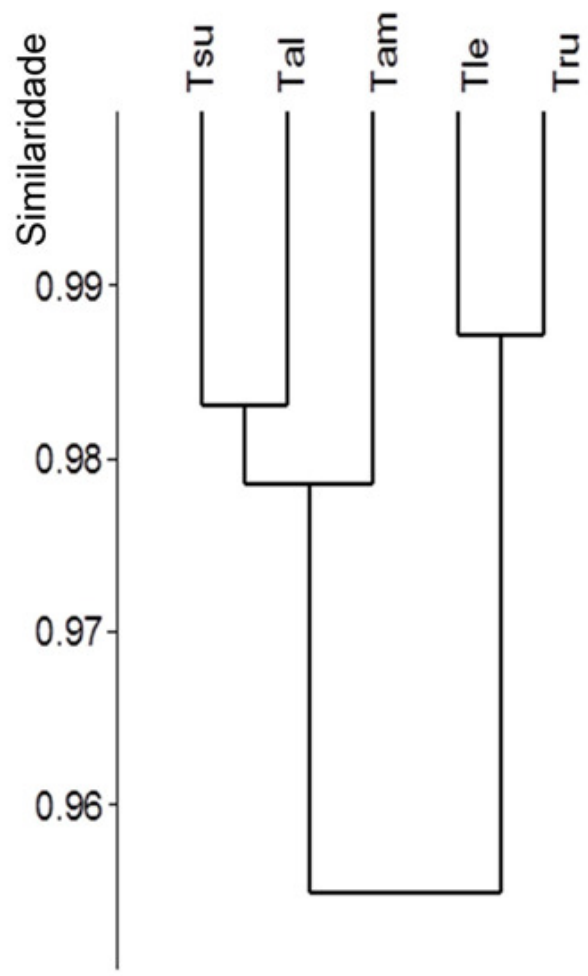

Figura 2. Ordenação resultante da análise de componentes principais (a) e dendrograma de similaridade entre espécies de turdídeos (b). Legendas: $($ quadrado e Tam $)=$ Turdus amaurochalinhus, $($ círculo e Tru $)=$ Turdus rufiventris, $($ triângulo e Tle $)=$ Turdus leucomelas, $(+\mathrm{e}$ Tal $)=$ Turdus albicollis $\mathrm{e}(\mathrm{x}$ e Tsu $)=$ Turdus subalaris.

\section{Discussão}

Entre as cinco espécies de turdídeos encontradas no Parque Municipal das Araucárias, a maior similaridade é observada para Turdus rufiventris e T leucomelas. A espécie T. rufiventris ocorre naturalmente em áreas abertas e fragmentos florestais, hábitats semelhantes aos de T. leucomelas (Collar 2005). Vogel et al. (2011) demonstraram regionalmente que a espécie de maior tamanho (T. rufiventris) apresentou maior variação morfológica do que T. leucomelas. Foi constatada, também naquele trabalho, maior amplitude de nicho trófico da primeira espécie em relação à segunda, o que garantiria uma melhor eficiência na competição interespecífica pelos recursos, sugerindo uma influência da morfologia em processos de competição interespecífica.

As espécies aqui estudadas têm sido apontadas como muito semelhantes morfologica (Sick 1997; Sigrist 2006) e funcionalmente em relação à guilda trófica do grupo (Clement 2000; Gasperin \& Pizo 2009). Sigrist (2006) afirma que a morfologia do gênero é uma convergência adaptativa à exploração do hábitat, compartilhada com outras famílias de aves. De modo geral, pequenas variações na forma entre táxons congenéricos sintópicos, mesmo que mínimas, permitem que elas coexistam (MacArthur \& Levins 1967), sen- do necessários estudos caso a caso para se testar esta hipótese (Vogel 2010).

O segundo agrupamento é formado por T. albicollis e $T$. subalaris, turdídeos com padrões de ocorrência muito variáveis, influenciados também por deslocamentos migratórios sazonais (Alves 2007). As espécies T. albicollis e $T$. subalaris co-ocorrem no local estudado apenas no verão (Vogel 2012), período durante o qual existe maior disponibilidade de recursos (Sick 1997; Ridgely \& Tudor 2001), atenuando efeitos de competição direta (Putmam 1996). Assim, tais espécies podem possuir estratégias de segregação sazonal, o que poderia ser interpretado como um mecanismo adicional de coexistência (Cody 1974). Estas informações são corroboradas por um estudo da estrutura da taxocenose e flutuação estacional da abundância nas capturas de turdídeos, que indicou maior intensidade da coocorrência das cinco espécies no verão e a coocorrência de apenas três delas (T. amaurochalinus, T. leucomelas e $T$. rufiventris) no inverno (Vogel et al. 2012).

Turdus albicollis depende de ambientes florestais (Collar 2005), o qual é compartilhado com T. subalaris. Contudo, esta última espécie ocupa estes hábitats somente durante migrações pelo Paraná, ocupando florestas ombrófilas e estacionais (Sigrist 2006).

Ridgely \& Tudor (2001) afirmam que T. amaurochalinus 
Tabela 2. Biometria de turdídeos do Parque Municipal das Araucárias, Guarapuava, Paraná. Média \pm desvio padrão, seguidos dos respectivos mínimos e máximos. a, b, c, d diferenças estatísticas através do teste post hoc LSD. * conjunto de dados heterocidásticos.

\begin{tabular}{|c|c|c|c|c|}
\hline $\begin{array}{c}\text { T. albicollis } \\
\text { Sabiá-coleira } \\
(\mathbf{N}=8)\end{array}$ & $\begin{array}{l}\text { T. amaurochalinus } \\
\text { Sabiá-poca } \\
(\mathbf{N}=41)\end{array}$ & $\begin{array}{c}\text { T. leucomelas } \\
\text { Sabiá-barranco } \\
(\mathbf{N}=46)\end{array}$ & $\begin{array}{c}\text { T. rufiventris } \\
\text { Sabiá-laranjeira } \\
(\mathrm{N}=51)\end{array}$ & $\begin{array}{c}\text { T. subalaris } \\
\text { Sabiá-ferreira } \\
(\mathbf{N}=3)\end{array}$ \\
\hline \multicolumn{5}{|c|}{ Massa (g) } \\
\hline $\begin{array}{c}60,78 \pm 2,99^{\mathbf{a}} \\
56,00-65,50 \\
\end{array}$ & $\begin{array}{c}61,69 \pm 4,10^{\mathbf{a}} \\
52,00-72,00 \\
\end{array}$ & $\begin{array}{c}72,79 \pm 4,01^{\mathbf{b}} \\
62,90-81,00 \\
\end{array}$ & $\begin{array}{c}73,04 \pm 4,01^{\mathbf{b}} \\
62,00-83,00 \\
\end{array}$ & $\begin{array}{c}61,43 \pm 2,10^{\mathrm{a}} \\
59,10-67,30 \\
\end{array}$ \\
\hline \multicolumn{5}{|c|}{ Comprimento total $(\mathrm{cm})$} \\
\hline $\begin{array}{c}22,37 \pm 0,69^{\mathrm{a}} \\
21,50-23,30\end{array}$ & $\begin{array}{c}22,81 \pm 0,84^{\mathrm{a}} \\
21,30-24,50\end{array}$ & $\begin{array}{l}23,68 \pm 0,16^{\mathbf{b}} \\
20,10-25,60\end{array}$ & $\begin{array}{c}24,40 \pm 0,14^{\mathbf{c}} \\
21,20-27,00\end{array}$ & $\begin{array}{c}21,07 \pm 0,41^{\mathbf{d}} \\
20,50-21,50\end{array}$ \\
\hline \multicolumn{5}{|c|}{ Comprimento do bico $(\mathrm{mm})$} \\
\hline $\begin{array}{c}27,07 \pm 0,46^{\mathrm{a}} \\
25,50-28,93\end{array}$ & $\begin{array}{c}26,18 \pm 0,20^{\mathrm{a}} \\
23,42-28,96\end{array}$ & $\begin{array}{c}26,64 \pm 0,32^{\mathbf{a}, \mathbf{b}} \\
15,41-30,49\end{array}$ & $\begin{array}{r}29,00 \pm 0,16^{\mathbf{b}} \\
25,10-31,47\end{array}$ & $\begin{array}{l}22,83 \pm 2,26^{*} \\
12,38-26,30\end{array}$ \\
\hline $\begin{array}{c}31,35 \pm 1,03^{\mathrm{a}} \\
29,00-32,33\end{array}$ & $\begin{array}{c}32,66 \pm 1,27^{\mathbf{b}} \\
29,00-35,00\end{array}$ & $\begin{array}{l}\text { Tarso }(\mathbf{m m}) \\
33,37 \pm 1,58^{\mathrm{c}} \\
30,70-39,00\end{array}$ & $\begin{array}{c}35,89 \pm 1,39^{\mathbf{d}} \\
32,00-38,73\end{array}$ & $\begin{array}{l}30,83 \pm 0,86^{\mathbf{a}} \\
29,84-32,90\end{array}$ \\
\hline $\begin{array}{r}107,75 \pm 4,49^{\mathbf{a}} \\
101,00-115,76\end{array}$ & $\begin{array}{r}111,85 \pm 4,56^{\mathbf{b}} \\
104,00-120,00\end{array}$ & $\begin{array}{c}\text { Asa (mm) } \\
115,13 \pm 3,89^{\mathbf{c}} \\
105,47-121,00\end{array}$ & $\begin{array}{r}115,10 \pm 4,18^{\mathfrak{c}} \\
102,00-128,00\end{array}$ & $\begin{array}{c}107,70 \pm 2,97^{\mathbf{a}, \mathbf{b}} \\
105,19-108,00\end{array}$ \\
\hline $\begin{array}{r}86,52 \pm 6,08^{\mathbf{a}} \\
74,00-94,04\end{array}$ & $\begin{array}{c}94,08 \pm 4,96^{\mathbf{b}} \\
82,00-105,00\end{array}$ & $\begin{array}{c}\text { Cauda }(\mathbf{m m}) \\
95,64 \pm 4,62^{\mathbf{b}} \\
83,00-104,00\end{array}$ & $\begin{array}{r}99,33 \pm 5,49^{\mathbf{c}} \\
84,63-107,00\end{array}$ & $\begin{array}{l}91,12 \pm 3,69^{\mathbf{a}, \mathbf{b}} \\
88,27-95,03\end{array}$ \\
\hline
\end{tabular}

ocorre com maior abundância em ambientes abertos. Dados recentes sugerem que esta espécie possui tanto populações residentes quanto migratórias (Capllonch et al. 2008), o que lhe confere um nicho intermediário entre os grupos formados pelos demais turdídeos estudados.

Comparações de massa e tamanho das espécies demonstraram métricas mais altas das aves neste trabalho, quando confrontados com os dados de Piratelli et al. (2001) para turdídeos do Mato Grosso do Sul, e de Roos et al. (2006) e Magalhães et al. (2007) para turdídeos de Pernambuco. Sugere-se deste modo, uma maior variação para o tamanho total de T. amaurochalinus, T. leucomelas e T. rufiventris.

Variações na massa das espécies capturadas neste estudo, em relação aos demais trabalhos, poderiam ser justificadas em função da concentração de capturas de turdídeos no PMA durante o período de outono e inverno (veja Vogel et al. 2011). Neste período, os indivíduos tendem a acumular gordura, preparando-se para o período invernal (King 1972). No entanto, esta hipótese não explica as variações encontradas para outros atributos biométricos como o comprimento. Outra hipótese é a de que as aves foram capturadas em ambiente urbano com grande quantidade de itens alimentares disponibilizados pela modificação do ambiente, como frutos de espécies exóticas amplamente utilizados por elas (Gasperin \& Pizo 2009). Entretanto, esta hipótese não explicaria a diferença no tamanho das estruturas. Adicionalmente, variações latitudinais na morfologia dessas aves poderiam ser interpretadas pela chamada Regra de Bergmann, onde existe uma tendência das espécies serem ligeiramente maiores em regiões mais frias do que em ambientes mais quentes, como forma de desenvolver uma menor relação entre superfície e volume corporal (Orr 1986; Blackburn \& Hawkins 2004).

\section{Conclusões}

Estudos visando avaliar a separação espacial e temporal podem ajudar a entender melhor a coexistência de espécies neotropicais de aves morfologicamente similares. Este estudo sobre a morfometria de cinco espécies da família Turdidae, quando relacionado à bioecologia dessas aves, sugere que tais espécies, consideradas morfologicamente semelhantes, podem estar segregadas espacial (T. leucomelas e T. rufiventris) ou temporalmente (T. subalaris, T. albicolis, e possivelmente $T$. amaurochalinus).

\section{Agradecimentos}

Agradecemos ao IBAMA, pela licença de pesquisa ( $\mathrm{n}^{\circ}$ 160531/ CEMAVE/SNA, processo $n^{\circ} 30311$ ), e ao MCNG e SEMAFLOR, pela autorização de pesquisa no Parque Municipal das Araucárias. À Coordenação de Aperfeiçoamento de Pessoal de Nível Superior (CAPES), pela bolsa de doutorado de HFV; além do Programa de Excelência Acadêmica (PROEX), pelo apoio técnico e financeiro ao Programa de Pós-graduação em Ecologia de Ambientes Aquáticos Continentais. Agradecimentos especiais a José Altair da Lúz, pelo auxílio em campo, e ao professor Luis Carlos Gomes, por sugestões nas análises estatísticas. 


\section{REFERÊNCIAS}

Alves, M.A.S. 2007. Sistemas de migrações de aves em ambientes terrestres no Brasil: exemplos, lacunas e propostas para o avanço do conhecimento. Revista Brasileira de Ornitologia 15(2): 231-238.

Auricchio, P. 2001. Aves. In: P. Auricchio \& M.G. Salomão (eds), Técnicas de Coleta e Preparação de Vertebrados. Instituto Pau Brasil História Natural, FAPESP, São Paulo, p. 127-148.

Blackburn, T.M. \& Hawkins, B.A. 2004. Bergmann's rule and the mammal fauna of northern North America. Ecography 27: 715-724.

Capllonch, P.; Ortiz, D. \& Soria, K. 2008. Migración del Zorzal común Turdus amaurochalinus (Aves, Turdidae) en Argentina. Revista Brasileira de Ornitologia 16(1): $12-22$.

CBRO (Comitê Brasileiro de Registros Ornitológicos) 2011. Listas das Aves do Brasil. 10 ed. Disponível em http:// www.cbro.org.br; acesso em 5 abr. 2012.

CEMAVE (Centro Nacional de Pesquisa e Conservação das Aves Silvestres) 2008. Manual de Anilhamento de Aves Silvestres. MMA, Brasília.

Clement, P. 2000. Thrushes. Princeton University Press, Princeton.

Cody, M.L. 1974. Competition and the Structure of Bird Communities. Princeton University Press, Princeton.

Collar, N.J. 2005. Family Turdidae (Thrushes). In: J. Del Hoyo, A. Elliot \& D.A. Christie (eds), Handbook of the Birds of the World. Vol. 10. Lynx Edicions, Barcelona.

Cordeiro, J. \& Rodrigues, W.A. 2007. Caracterização fitossociológica de um remanescente de Floresta Ombrófila Mista em Guarapuava, PR. Revista Árvore 31(3): 545-554.

Enout, A.M.J.; Lobato, D.N.; Azevedo, C.S. \& Antonini, Y. 2009. Parasitismo por malófagos (Insecta) e ácaros (Acari) em Turdus leucomelas (Aves) nas estações reprodutivas e de muda de penas no Parque Estadual do Rio Preto, Minas Gerais, Brasil. Zoologia 26(3): 534 540.

Gasperin, G. \& Pizo, M.A. 2009. Frugivory and habitat use by Thrushes (Turdus spp.) in a suburban area in south Brazil. Urban Ecosystems 12(1): 425-436.

Hammer, Ø.; Harper, D.A.T. \& Ryan, P.D. 2001. PAST: Paleontological Statistics Software Package for Education and Data Analysis. Palaeontologia Electronica 4(1): 19. Disponível em http://palaeo-electronica.org/2001_1/ past/issue1_01.htm; acesso em 5 abr. 2012.

King. J.R. 1972. Adaptive periodic fat storage by birds. Proceedings of International Ornithology Congress 15(1): 200-217.

Leite, A.G.; Barreiros, M.H.M.; Cunha, J.G. \& Brito, R.D.S. 2010. Predação do sabiá-laranjeira Turdus rufiventris (Passeriformes: Turdidae) por tucano-de-bicoverde Ramphastos dicolorus (Piciformes: Ramphastidae) no município de Campos do Jordão, SP / Brasil. Atualidades Ornitológicas 158(3): 55-56.

MacArthur, R. \& Levins, R. 1967. The limiting similarity, convergence, and divergence of coexisting species. The American Naturalist 101(921): 377-385.

Magalhães, V.S.; Azevedo-Júnior, S.M.; Lyra-Neves, R.M.; Telino-Júnior, W.R. \& Souza, D.P. 2007. Biologia de aves capturadas em um fragmento de Mata Atlân- tica, Igarassu, Pernambuco, Brasil. Revista Brasileira de Zoologia 24(4): 950-964.

O’Neill, J.O.; Lane, D.F. \& Naka, L.N. 2011. A cryptic new species of thrush (Turdidae: Turdus) from western Amazonia. The Condor 113(4): 869-880.

Orr, R. T. 1986. Biologia dos Vertebrados. Roca, São Paulo.

Piratelli, A.; Siqueira, M.A.C. \& Marcondes-Machado, L.O. 2001. Reprodução e muda de penas em aves de sub-bosque na região leste de Mato Grosso do Sul. Ararajuba 8(2): 99-107.

Putmam, R. J. 1996. Community Ecology. Chapman \& Hall, London.

Ridgely, R.S. \& Tudor, G. 2001. The Birds of South America. Vol. 2. The Oscine Passerines. University of Texas Press, Austin.

Roos, A.L.; Nunes, M.F.C.; Sousa, E.A.; Sousa,A.E.B.A.; Nascimento, J.L.X. \& Lacerda, R.C.A. 2006. Avifauna da região do Lago de Sobradinho: composição, riqueza e biologia. Ornithologia 1(2): 135-160.

Sazima, I. \& D'Angelo, B.G. 2011. The Pale-breasted Thrush (Turdus leucomelas) preys on a gekkonid lizard and an anomalepidid snake. Revista Brasileira de Ornitologia 19(3): 450-452.

Sick, H. 1997. Ornitologia Brasileira. Nova Fronteira, Rio de Janeiro.

Sigrist, T. 2006. Aves do Brasil. Uma visão artística. Fosfértil, São Paulo.

Storni, A.; Alves, M.A.S. \& Valim, M.P. 2005. Ácaros de penas e carrapatos (Acari) associados à Turdus albicollis Vieillot (Aves, Muscicapidae) em uma área de Mata Atlântica da Ilha Grande, Rio de Janeiro, Brasil. Revista Brasileira de Zoologia 22(2): 419-423.

Straube, F. C. \& Bianconi, G. V. 2002. Sobre a grandeza e a unidade utilizada para estimar o esforço de captura com a utilização de redes-de-neblina. Chiroptera Neotropical 8(2): 150-152.

Thomaz, E. L. \& Vestena, L. R. 2003. Aspectos Climáticos de Guarapuava-PR. Editora da Unicentro, Guarapuava.

Vogel, H.F. 2010. Comunidade e Partilha Ecológica de Turdídeos (Aves: Passeriformes) em um Fragmento Urbano de Floresta com Araucárias em Guarapuava no Sul do Brasil. Dissertação (Mestrado em Ciências Biológicas). Universidade Estadual do Centro-Oeste.

Vogel, H.F. 2012. Thrushes: reasons for their use in animal ecology studies. SaBios 7(1): 66-69.

Vogel, H.F.; Zawadzki, C.H. \& Metri, R. 2011. Partilha ecológica entre Turdus leucomelas Vieillot, 1818 e Turdus rufiventris Vieillot, 1818 (Aves: Passeriformes) em um fragmento urbano de Floresta com Araucárias, Sul do Brasil. Biota Neotropica 11(3): 35-45.

Vogel, H.F., Zawadzki, C.H. \& Metri, R. 2012. Occurrence of thrushes inan urban fragment of Araucaria forest in southern Brazil. Biota Neotropica 12(4): 242-247

Wiens, J.A. 1982. On size ratios and sequences in ecological communities: Are there no rules? Annals Zoologies Fennici 19: 297-308. 